\title{
LOS GENES COMO MARCADORES DE RIESGO EN CÁNCER GÁSTRICO: REVISIÓN DE ESTUDIOS EN POBLACIÓN COLOMBIANA
}

\section{GENES AS RISK MARKERS IN GASTRIC CANCER: REVIEW OF STUDIES IN COLOMBIAN POPULATION}

\begin{abstract}
Forma de citar: YÁÑEZ-GUTIÉRREZ Claudia. Los genes como marcadores de riesgo en cáncer gástrico: Revisión
\end{abstract} de estudios en población colombiana. Respuestas. 2013; 18(2): 61-73.

Recibido:

Julio 3 de 2013

Aceptado:

Octubre 25 de 2013
${ }^{1}$ Magister en Biología. Universidad de Santander genesis.cuc@campus.udes.edu.co Cúcuta - Colombia.

\author{
Claudia Marcela Yáñez-Gutiérrez $z^{1}$
}

\section{RESUMEN}

El objetivo de esta revisión, fue identificar el rol de los genes como marcadores de riesgo en cáncer gástrico (CG) en población colombiana. Se revisaron publicaciones de investigaciones realizadas en los últimos diez años, utilizando las bases MEDLINE y LILACS y complementando la pesquisa con la bibliografía relevante de los artículos. Se encontraron estudios en busca de asociación de CG con polimorfismos de varios genes humanos involucrados en la respuesta inmune, la desintoxicación y el supresor p53. En Colombia al igual que en otros países, las evidencias de asociación de polimorfismos genéticos con CG son aún controversiales, debido a la variación de los resultados que arrojan los estudios en las diferentes poblaciones. El genoma de las cepas de Helicobacter pylori que infectan población colombiana también ha sido investigado en búsqueda de polimorfismos de virulencia. El genotipo cagA/vacAs $1 m 1$ identificado como citotóxico en esta bacteria, mostró en la mayoría de las investigaciones, asociación con CG. La evidencia de asociación de CG con factores genéticos en población colombiana no es concluyente. Está lejos aún, la identificación de marcadores genéticos que permitan predecir el riesgo a desarrollar CG. A pesar de ello, algunos polimorfismos de genes humanos como los de IL-1 o los de algunas enzimas desintoxicantes, así como los genes cagA y vacA de Helicobacter pylori podrían ser candidatos a futuros marcadores de riesgo en esta neoplasia.

Palabras clave: cáncer gástrico, riesgo, genotipo, Colombia

\section{ABSTRACT} 0122-820X

The objective of this review was to identify the role of genes as risk markers in gastric cancer (GC) in Colombian population studies. The study reviewed research publications in the last ten years, using the MEDLINE and LILACS, as well as various literature research of relevant articles. Searching studies found GC association with several human 
gene polymorphisms involved in the immune response, detoxification and suppressor p53. In Colombia, as in other countries, the evidence of the association of genetic polymorphisms with GC are still controversial because of the variation in results that studies in different populations. The genome of Helicobacter pylori strains that infect Colombian population has also been investigated in search of polymorphisms of virulence. cagA/ vacAs $1 \mathrm{~m} 1$ genotype identified as cytotoxic in this bacterium, demonstrated most of the research associated with GC. Evidence of association of GC with Colombian population genetic factors was inconclusive. It is yet to be determined the exact identification of genetic markers that can predict the risk of developing GC. However, some human gene polymorphisms as IL-1 or some detoxifying enzymes and the vacA and cagA of $H$. pylori could be candidates for future risk markers in these tumors.

Keywords: gastric cancer, risk, genotype, Colombia

\section{INTRODUCCIÓN}

$\mathrm{E}^{\mathrm{n}}$ n Colombia, el cáncer gástrico es la primera causa de muerte por tumores malignos y el tercero en incidencia, teniendo en cuenta ambos sexos $(1,2)$, por lo cual esta neoplasia se ha convertido para el país, en un problema de salud pública con fuerte impacto a nivel social y económico. Los departamentos con mayor carga de la enfermedad son aquellos ubicados en regiones montañosas de la geografía colombiana en contraste con los de zonas costeras (3). La diferencia en la incidencia entre poblaciones, puede ser dada por variaciones en la susceptibilidad genética y la virulencia de las cepas de Helicobacter pylori circulantes, los factores ambientales y aquellos relacionados con el estilo de vida. Para enfrentar esta problemática, el Plan Nacional para el Control del Cáncer, propone, el control del riesgo y la detección temprana con el fin de disminuir la carga de la enfermedad (4). En este sentido, cobra importancia la búsqueda de biomarcadores que permitan identificar aquellos individuos de mayor riesgo dentro de una población, con el fin de implementar estrategias de prevención. El propósito de esta revisión, fue identificar los avances de las investigaciones para la detección de marcadores genéticos asociados con esta neoplasia en población colombiana.

\section{MATERIALES Y MÉTODOS}

Esta revisión presenta estudios de los últimos diez años en busca de asociación de polimorfismos humanos y marcadores de virulencia de Helicobacter pylori con cáncer gástrico en población colombiana. Se revisó la literatura con el fin de responder icuáles son los marcadores genéticos asociados a cáncer gástrico en población colombiana? Se utilizaron las bases MEDLINE y LILACS, usando como palabras clave "cáncer gástrico, polimorfismos, H. pylori y Colombia” y complementando la pesquisa con la bibliografía relevante de los artículos. En cada estudio se tuvo en cuenta, la población, diseño del estudio, tamaño de la muestra, resultados, significancia estadística y discusión.

\section{RESULTADOS Y DISCUSIÓN}

\section{Los genes humanos}

En este aspecto, las investigaciones, dirigen sus esfuerzos a la búsqueda de asociación de polimorfismos genéticos o variantes alélicas con capacidad de hacer susceptible a su portador al desarrollo de cáncer gástrico (CG). En Colombia se han explorado principalmente algunos polimorfismos en los genes de Interleuquina-1 $\beta$ (IL-1 $\beta$ ), Interleuquina-1Ra 
(receptor antagónico), Interleuquina-10 (IL10), Factor de Necrosis Tumoral- $\alpha$ (FNT- $\alpha$ ), gen supresor $p 53$ y los genes de enzimas Glutatión S-Transferasas (GST) y Citocromo P450 (CYP).

Polimorfismos en genes IL-1 $\beta$ e IL-1Ra. La inflamación crónica, desencadenada por la respuesta inmune a la infección por Helicobacter pylori, ha sido identificada en algunos estudios como un evento previo a lesiones pre-malignas en la mucosa gástrica. Existen diferencias interindividuales en esta respuesta debido a la presencia de variantes alélicas en los genes involucrados. La IL-1 $\beta$ es una citoquina pro-inflamatoria que junto con la citoquina anti-inflamatoria IL-1Ra participa en la respuesta inducida por la infección de $H$. pylori. Algunos polimorfismos en las posiciones $-511,-31,+3954$ del gen IL-1 $\beta$ han sido especialmente asociados con CG (5). Los genotipos IL-1 $\beta-511 \mathrm{~T} / \mathrm{T}$ e IL-1RN A2/A2 han sido asociados con mayor producción de IL-1 $\beta$ y con una fuerte respuesta inflamatoria en la mucosa gástrica y mayor riesgo de enfermedad $(6,7)$. La búsqueda de asociación de estos dos genotipos con CG, ha sido abordada en población colombiana por Martínez et al. (8) con pacientes de las ciudades de Tunja y Bogotá, con alto riesgo para esta neoplasia. El genotipo más frecuente en las dos poblaciones tanto para casos como para controles fue IL$1 \beta-511 \mathrm{C} / \mathrm{T}$. La asociación del polimorfismo IL-1 $\beta$-511T/T con CG solo se evidenció en pacientes de Tunja (Tabla 1). El polimorfismo $1 R A * 2$ solo mostró asociación en pacientes seropositivos para anticuerpos IgG anti CagA de la ciudad de Tunja (Tabla 1). En otro estudio, Martínez et al. (9) compararon pacientes con CG y úlcera duodenal de estas dos ciudades con pacientes de ciudades costeras con bajo riesgo para esta neoplasia (Barranquilla, Cartagena y Santa Marta), evidenciando asociación del genotipo IL-1 $\beta-511 \mathrm{~T} / \mathrm{T}$ con CG tanto en los pacientes de Tunja como en los de Bogotá (Tabla 1). El polimorfismo -511 fue estudiado junto con los polimorfismos -31 , +3954 en pacientes con patología gástrica diversa (gastritis crónica no atrófica, úlcera gástrica, gastritis atrófica, metaplasia, displasia y cáncer) de la ciudad de Ibagué (Tolima), sin hallazgos de asociación de ninguno de estos polimorfismos con el desarrollo de patología no benigna $(10,11)$.

Los estudios en busca de asociación entre CG y el polimorfismo IL-1 $\beta-511 \mathrm{~T} / \mathrm{T}$ arrojan resultados contradictorios no solo entre las poblaciones colombianas estudiadas (Tunja, Bogotá e Ibagué), también al ser comparados con los resultados de un estudio realizado por Cañas et al. (12) en población de alto riesgo para CG del centro-occidente de Venezuela, donde no hubo evidencia de asociación de esta neoplasia con el genotipo IL-1 $\beta-511 \mathrm{~T} / \mathrm{T}$ pero si con el IL-1RN A2/A2. El incremento del riesgo para CG encontrado por Martínez et al. $(8,9)$ en portadores del alelo IL-1RN*A2 y seropositivos para anticuerpos anti CagA, podría ser un indicio de cómo la interacción entre dos o más factores inciden en el aumento del riesgo para esta enfermedad. En esta revisión sólo fue hallado un estudio colombiano buscando asociación de CG con los polimorfismos - 31 y +3954 , sin evidencia de la misma.

Polimorfismos en el gen del FNT- $\alpha$. Este factor tiene función pro-inflamatoria y sus niveles se encuentran alterados en varias enfermedades, incluyendo pacientes con infección por $H$. pylori. Una variante del alelo de referencia para esta citoquina se origina por la sustitución de Guanina $(\mathrm{G})$ por Adenina (A) en la posición -308 del promotor de este gen (13). Su presencia se manifiesta con niveles elevados de FNT- $\alpha$ y ha sido asociado con el riesgo para CG (14). En Colombia, el estudio de este polimorfismo, ha sido abordado en pacientes de las ciudades de Popayán, Bogotá, Tunja, Barranquilla, Santa Marta y Cartagena, (siendo las tres primeras, zonas de alto riesgo para $\mathrm{CG}$ ), sin encontrar evidencia de su asociación con CG $(9,15)$. Ambos estudios reportan frecuencia casi nula del genotipo homocigoto (A/A) para este gen. La baja frecuencia de este polimorfismo en población colombiana es similar a la reportada en población asiática y contraria a los hallazgos en poblaciones caucásicas, en las cuales hay evidencias de asociación de este polimorfismo con CG (16).
Junio - Diciembre 2013 ISSN 0122-820X PP: 61-73 
No. 2

Junio - Diciembre 2013 ISSN 0122-820X

PP: 61-73
Polimorfismos del gen IL-10. Los polimorfismos -819 y -1082 de interleuquina-10 fueron indagados en población de Tunja y Bogotá, encontrando predominio del genotipo IL-10$1082 \mathrm{~A} / \mathrm{A}$, sin hallazgos de asociación con CG (9).

Polimorfismo en el codón 72 del gen $p 53$. El p53 es un gen supresor de tumores, llamado el "guardián del genoma", codifica para una proteína de $53 \mathrm{Kd}$ con la función de incapacitar a la célula para evitar la división celular o activar la apoptosis frente a injurias en el ADN. En el codón 72 ubicado en el exón cuatro de este gen, se tiene identificada una mutación que da lugar en la proteína al cambio de Arginina (Arg) por Prolina (Pro). El alelo Pro codifica una proteína que presenta deficiencias en su función, lo que facilita acumulación de mutaciones en la célula, promoviendo la presencia de neoplasias. Este polimorfismo ha sido asociado no solo con CG sino también con cáncer de colon y de glándula mamaria (17). En Colombia, fue estudiado por Cardona et al. (18) en pacientes afectados por neoplasia gástrica primaria provenientes de la ciudad de Manizales, encontrando mayor frecuencia del alelo Arg, considerado el normal, sin evidencia de asociación del alelo Pro con la enfermedad. La escasa frecuencia del alelo Pro en este estudio, coincide con lo reportado por Zhou et al. para población caucásica (19). Shepherd et al. (17), reportan mayor frecuencia de este alelo en individuos de raza negra. La búsqueda de asociación de este polimorfismo arroja resultados contradictorios tanto para población asiática como caucásica (17, $19,20)$.

Polimorfismos en genes de enzimas GST. Las enzimas GST intervienen en la desintoxicación de carcinógenos químicos. Varios estudios han reportado susceptibilidad a CG y de colon en individuos que poseen el genotipo homocigoto nulo para la expresión de estas enzimas (21-24). Torres et al. (15) estudiaron en pacientes de la ciudad de Popayán, variantes genéticas de GSTM1 y GSTT1. La prevalencia de GSTM1-0 (polimorfismo nulo) fue alta en los pacientes con CG $(65,2 \%)$ al ser comparada con la del grupo control $(37,5 \%)$, permitiendo a los investigadores establecer asociación entre este polimorfismo y la enfermedad (Tabla 1). El polimorfismo GSTT1-0 no mostró diferencias. Castaño-Molina et al. (25) por su parte, en población de Manizales, no encontraron asociación entre CG y GSTM1-0 pero si con el polimorfismo GSTT1-0 al igual que con el GSTP1/val, siendo este último, asociado también con riesgo para el desarrollo de cáncer.

La asociación de los polimorfismos de los genes de enzimas GST con CG muestra resultados controversiales no solo entre poblaciones de Colombia (Popayán y Manizales), sino entre diferentes regiones del mundo (21-24).

Polimorfismos en genes de la familia Citocromo P450 (CYP). La Citocromo P450 agrupa enzimas, requeridas en el metabolismo de tóxicos y fármacos. Las enzimas CYP1A1 y CYP1A2 participan en la activación de numerosos compuestos procarcinógenos, convirtiéndolos en metabolitos reactivos con facultad para desencadenar cáncer. Los alelos CYP1A1*2A y CYP1A1*2C han sido asociados con aumento del riesgo de neoplasias (26). Castaño -Molina et al. (27) en un estudio realizado en el departamento de Caldas, demostraron la asociación de CG con el alelo C2 de CYP2E1*5A (tabla 1). En contraste, los alelos CYP1A1*2A y CYP1A1*2C no mostraron diferencias significativas entre los grupos caso y control. La presencia simultánea del alelo C2 y el hábito de fumar, aumenta la asociación con CG, ocurriendo lo mismo con el alelo CYP1A1*2C (Tabla 1). Estos últimos resultados resaltan la importancia de la interacción entre genes y ambiente para la expresión del fenotipo.

Alelo A del sistema sanguíneo ABO. Muchos microorganismos poseen como parte de su mecanismo de colonización e infección, la adherencia a carbohidratos presentes en la célula hospedera, siendo el Helicobacter pylori uno de ellos. Löfling et al. (28) comprobaron la afinidad de esta bacteria al antígeno Lewis b, quedando claro, que la expresión de este grupo sanguíneo facilita la colonización de 
células por la bacteria, promoviendo el desarrollo de la enfermedad. La expresión del antígeno A del sistema sanguíneo $\mathrm{ABO}$ al igual que el antígeno Lewis b, se expresa en células del epitelio gástrico. En Colombia, un estudio llevado a cabo por Bermúdez et al. (29) con población del departamento de Santander, encontró evidencia de asociación entre CG y el grupo sanguíneo $\mathrm{A}(\mathrm{p}=0,001)$ (Tabla 1). Esta asociación se ha indagado también en población de Asia y de Brasil, con resultados aún controversiales $(30,31,32)$.

\section{Los genes de Helicobacter pylori}

Diez años después del descubrimiento de Helicobacter pylori por Warren y Marshall (1983), la Agencia Internacional para Investigación en Cáncer (IARC), determinó la infección por esta bacteria, como causa principal de CG (33). Sin embargo, aunque más de la mitad de la población mundial está infectada con $\mathrm{H}$. pylori, sólo un pequeño porcentaje de individuos infectados, desarrollan la enfermedad, haciendo suponer, la presencia de factores bacterianos asociados a su capacidad de virulencia. Es por ello que las investigaciones actuales, pretenden establecer marcadores de virulencia que permitan identificar las cepas de esta bacteria, con potencial cancerígeno.

Gen cagA y vacA. Es bien conocida la importancia del gen cagA al codificar una proteína (CagA) capaz de producir alteraciones del citoesqueleto de células epiteliales $(34,35)$ y generar fuerte actividad pro inflamatoria y anti-apoptosis (36), induciendo lesiones precancerosas que pueden desencadenar en CG (37). El vacA, es otro gen señalado como posible implicado en la virulencia de $H$. pylori. La toxina VacA forma vacuolas intracitoplasmaticas y poros en la membrana celular de células epiteliales, facilitando la salida de urea, que al ser hidrolizada por la ureasa de $\mathrm{H}$. pylo$r i$, permite la sobrevivencia de la bacteria en el ambiente gástrico. Esta toxina al igual que CagA, genera cambios en la estructura de la célula epitelial. Contrario a cagA, el gen vacA ha sido encontrado en todas las cepas de $\mathrm{H}$. pylori. Este gen, se divide en dos regiones (s y m) y según las variaciones (polimorfismos) que presentan, pueden ser clasificadas en "s1" o "s2" y "m1" o "m2". Las cepas vacAs $1 \mathrm{~m} 1$ poseen mayor actividad citotóxica que las cepas vacAs2m2 (38). Lo anterior, permite identificar como cepas citotóxicas, aquellas que poseen el genotipo vacAs $1 \mathrm{~m} 1 / \mathrm{cag} A+/ \mathrm{cagE}+$.

Otros genes relacionados con la capacidad de virulencia de $H$. pylori, son el oipA, que codifica una proteína capaz de despertar la respuesta proinflamatoria de la IL-8 en el huésped (39) y el babA2, determinante de la adhesina BabA2 con afinidad por el antígeno Lewis b expresado en las células del epitelio gástrico (40). Estos genes también han sido objeto de estudio en población colombiana.

En Colombia, los marcadores de virulencia de H. pylori han sido estudiados en cepas aisladas de población con patología gástrica diversa. Citelly et al. (41) hallaron alta frecuencia de los genes cagA, vacA e iceA, y predominio del genotipo cagA/vacAs $1 m 1$ en pacientes con patología severa (ulcera péptica, metaplasia intestinal y adenocarcinoma gástrico) $(\mathrm{p}=0,009)$. Quiroga et al. (42) reportaron frecuencia de 63,7\% para el genotipo citotóxico vacAs $1 \mathrm{~m} 1 /$ $c a g A+/ c a g E+$ en pacientes con enfermedad gástrica, siendo más frecuente (84\%) en pacientes con CG (Tabla2). No encontraron asociación de los genes oipA y babA2 con la severidad de la enfermedad a pesar de su alta frecuencia en cepas citotóxicas. Los hallazgos con el gen babA2 en población colombiana son similares a los de Mizushima et al. (43) en población japonesa pero contradictorios con lo encontrado en población Alemana (40). López et al. (44) al estudiar el gen vacA en cepas de pacientes del departamento del Tolima halló $52 \%$ de frecuencia del genotipo vacA/ slml, sin demostrar asociación de este genotipo con CG o lesiones precancerosas. Loh et al. (45) por su parte, investigaron la variación en la expresión de la proteína CagA en cepas de H. pylori aisladas de pacientes del departamento de Nariño, demostrando que las cepas provenientes de Túquerres, en las montañas andinas (con alto riesgo para CG), expresan altos niveles de la toxina, en contraste con los aislados de Tumaco, en la costa pacífica (de
ISSN 0122-820X

PP: 61-73 
No. 2

Junio - Diciembre 2013 ISSN 0122-820X

PP: 61-73 bajo riesgo). Los altos niveles de expresión de CagA fueron asociados con la presencia de una secuencia específica (AATAAGATA), localizada hacia el extremo 5'del gen (Tabla 2). El estudio filogeográfico de estas cepas, permitió identificar el origen europeo de las cepas de Túquerres y el origen africano en las de Tumaco.

De los anteriores estudios se deduce que los genes cagA y vacAs $1 \mathrm{~m} 1$ tienen potencial como marcadores de virulencia y de riesgo incrementado a desarrollar patología gástrica severa en población colombiana, aunque es importante continuar con los estudios de virulencia específicos para las cepas circulantes en cada población con riesgo. El trabajo de Loh et al. resalta la importancia de cagA como marcador de virulencia mostrando evidencia de la variación en la expresión de CagA y el valor de la identificación de nuevos marcadores intragénicos como la secuencia AATAAGATA, que puedan asociarse a severidad de la patología gástrica.

Secuencia EPIYA en la proteína CagA. Los hallazgos de cepas de $\mathrm{H}$. pylori $\mathrm{CagA}+$ con diferente nivel de asociación con CG, ha inducido al estudio de los polimorfismos de esta proteína. Al respecto, se ha identificado la región EPIYA. Esta región es el blanco de la fosforilación que sufre CagA por las quinasas del huésped. En la actualidad se han descrito cuatro tipos de secuencias EPIYA (A, B, C y D) (46) con variaciones en el número de repeticiones entre las diferentes cepas. La virulencia y la incidencia de CG han sido asociadas a un mayor número de repeticiones $(47,48)$. En población colombiana, Schneider, et al. (49) encontraron relación entre el número de secuencias EPIYA con el CG $(p<0.001)$ (Tabla 2). Quiroga et al. (50), hallaron asociación entre CG y la infección con cepas de H. pylori que poseen tres o más repeticiones EPIYA-C. Por el contrario, Fajardo et al. (51), también en población colombiana, describen la falta de asociación entre las variaciones EPIYA con $\mathrm{CG}$, siendo coincidente este resultado con un hallazgo de Torres (52) en población venezolana. El número de motivos EPIYA se relaciona con el número de sitios de fosforilación de la proteína CagA y con un mayor porcentaje de rearreglos del citoesqueleto (elongaciones) producidas por la bacteria en las células epiteliales gástricas. Varios estudios reportan que cepas con mayor número de motivos EPIYAC sufren mayor fosforilación de CagA, por lo que sus efectos celulares son más intensos. Pudiéndose deducir que el número de secuencias EPIYA-C podría considerarse como determinante de virulencia de las cepas de $\mathrm{H}$. pylori $(49,50)$, aunque los resultados siguen siendo controversiales $(51,52)$.

Otro genes de H. pylori. Los genes de la familia hom que codifican para proteínas externas de membrana, también son investigados como posibles determinantes de virulencia. Algunos estudios sugieren que el producto del gen homB podría estar asociado con la presencia de ulcera gástrica $(53,54)$. Jung et al. $(55)$ reportan alta frecuencia de homB en pacientes con CG provenientes de población colombiana y anglosajona (Tabla 2). La familia de genes jhp fue estudiada por Watada et al. (56) quienes al analizar cepas de $H$. pylori cagA+ de pacientes colombianos y japoneses con enfermedad gástrica, encontraron alta prevalencia de los genes jhp0045 - jhp0046 en pacientes colombianos. Aún son pocas las evidencias de asociación de estos genes con CG para conocer su utilidad como marcadores.

\section{CONCLUSIONES}

Los estudios de asociación de CG con variaciones alélicas en genes humanos, son aún contradictorios. En esta revisión, se encontró evidencia de asociación del polimorfismo IL-1 $\beta-511 \mathrm{~T} / \mathrm{T}$ y los de genes de las enzimas GST y CYP, en algunas poblaciones colombianas. Los resultados contradictorios pueden ser producto del reducido número de muestra utilizado en algunas investigaciones y de la variación de las frecuencias genotípicas en las poblaciones. El análisis combinado de variables enzimáticas y los tóxicos endógenos o exógenos sobre los cuales ellas actúan, podría permitir evidenciar de manera más clara el riesgo que se quiere demostrar. También es 
necesario, abrir la posibilidad al análisis de otros biomarcadores como los aductos y las aberraciones cromosómicas. Respecto al genoma de H. pylori, los hallazgos muestran alta frecuencia de cepas cagA + en la población de Colombia y asociación del genotipo citotóxico $c a g A / v a c A m 1 s 1$ con el riesgo a CG. La región EPIYA al igual que los genes de la familia hom y jhp tienen potencial para convertirse en otros marcadores de riesgo; para averiguarlo, son necesarios más estudios.

\section{AGRADECIMIENTOS}

Este trabajo fue posible gracias al apoyo de la Universidad de Santander (UDES), y al grupo de investigación Génesis del programa de Bacteriología y Laboratorio Clínico, sede Cúcuta.

Tabla 1. Polimorfismos de genes humanos asociados a cáncer gástrico en población colombiana.

\begin{tabular}{|c|c|c|c|c|c|}
\hline Polimorfismo & ID gen & Población & $\begin{array}{l}\text { Tipo de } \\
\text { estudio }\end{array}$ & $\begin{array}{c}\text { Asociación } \\
\text { OR (IC 95\%) }\end{array}$ & Referencia \\
\hline$I L-1 \beta-511 T / T$ & 3553 & $\begin{array}{c}\text { Tunja } \\
\text { Tunja, Bogotá, } \\
\text { Barranquilla, } \\
\text { Cartagena, } \\
\text { Santa Marta } \\
\end{array}$ & $\begin{array}{l}\text { Casos y } \\
\text { controles }\end{array}$ & $\begin{array}{c}11,31 \\
1,20-106,54 \\
\\
\\
4,69 \\
1,22-18,09\end{array}$ & 8 \\
\hline $\begin{array}{l}\text { IL-1RNA*2 e infección } \\
\text { con } H \text {. pylori CagA+ }\end{array}$ & 3557 & Tunja & $\begin{array}{l}\text { Casos y } \\
\text { controles }\end{array}$ & $\begin{array}{c}14,83 \\
1,41-155,89 \\
\end{array}$ & 8 \\
\hline $\begin{array}{c}\text { Polimorfismo nulo } \\
\text { GSTMI }\end{array}$ & 2944 & Cauca & & $\begin{array}{c}* * 5,45 \\
1,72-17,20\end{array}$ & 15 \\
\hline $\begin{array}{l}\text { Polimorfismo nulo } \\
\text { GSTM1+tabaquismo }\end{array}$ & & Caldas & $\begin{array}{l}\text { Casos y } \\
\text { controles }\end{array}$ & $\begin{array}{c}3,0 \\
1,2-7,4\end{array}$ & 25 \\
\hline $\begin{array}{l}\text { Polimorfismo nulo } \\
\text { GSTT1 } \\
\text { Polimorfismo nulo } \\
\text { GSTT1+tabaquismo }\end{array}$ & 2952 & Caldas & $\begin{array}{l}\text { Casos y } \\
\text { controles }\end{array}$ & $\begin{array}{c}* 2,5 \\
1,2-4,9 \\
4,9 \\
1,8-13,2\end{array}$ & 25 \\
\hline $\begin{array}{c}\text { Alelo GSTP1/val } \\
\text { Alelo } \\
\text { GSTP1/val+tabaquismo }\end{array}$ & 2950 & Caldas & $\begin{array}{l}\text { Casos y } \\
\text { controles }\end{array}$ & $\begin{array}{c}* 1,9 \\
1,03-3,7 \\
4,2 \\
1,7-10,5\end{array}$ & 25 \\
\hline $\begin{array}{l}\text { Alelo } C 2 C Y P 2 E 1 * 5 A \\
\text { Alelo } C 2 C Y P 2 E 1 * 5 A \\
+ \text { tabaquismo }\end{array}$ & 1571 & Caldas & $\begin{array}{l}\text { Casos y } \\
\text { controles }\end{array}$ & $\begin{array}{c}* 3,6 \\
1,6-8,1 \\
\\
\\
5,3 \\
2,0-14,1\end{array}$ & 27 \\
\hline $\begin{array}{c}\text { Alelo } C Y P 1 A 1^{*} 2 C+ \\
\text { tabaquismo }\end{array}$ & 1543 & Caldas & $\begin{array}{l}\text { Casos y } \\
\text { controles }\end{array}$ & $\begin{array}{c}2,7 \\
1,2-6,2\end{array}$ & 27 \\
\hline Alelo A sistema $\mathrm{ABO}$ & 28 & Santander & $\begin{array}{l}\text { Casos y } \\
\text { controles }\end{array}$ & $\begin{array}{c}2,22 \\
1.38-3.57\end{array}$ & 29 \\
\hline
\end{tabular}

*OR e IC 95\% ajustado por edad, sexo y tabaquismo

** OR e IC 95\% ajustado por edad, sexo, educación, familia con cáncer, ingestión de comida ahumada, consumo de cigarrillo, consumo de alcohol e infección por H. pylori. 
Tabla 2. Polimorfismos genéticos de Helicobacter pylori asociados a cáncer gástrico en población colombiana.

\begin{tabular}{|c|c|c|c|}
\hline Polimorfismo & Población & Asociación & Referencia \\
\hline \multirow{3}{*}{$\operatorname{cag} A+$} & $\begin{array}{l}\text { Tunja } \\
\text { Bogotá }\end{array}$ & $\begin{array}{c}\mathrm{OR}=11,56 \\
2,62-50,91 \\
\mathrm{OR}=19,66 \\
0,98-395,70\end{array}$ & 8 \\
\hline & Tunja-Bogotá & $\begin{array}{l}\mathrm{OR}=4,43 \\
1,72-11,41\end{array}$ & 9 \\
\hline & $*$ se & $\mathrm{p}=0,016$ & 55 \\
\hline vacA sl & $*$ se & $\mathrm{p}=0,005$ & 41 \\
\hline vacA $m 1$ & *se & $\mathrm{p}=0,004$ & 41 \\
\hline vacAsIm $1 / \mathrm{cag} A+/ \mathrm{cag} E+$ & $*$ se & $\mathrm{P}=0,002$ & 42 \\
\hline $\begin{array}{l}\text { Secuencia } \text { AATAAGATA } \\
\text { localizada } 40 \text { nucleótidos } \\
\text { rio arriba del inicio de la } \\
\text { transcripción de } \operatorname{cag} A \text {. }\end{array}$ & $\begin{array}{c}\text { Nariño } \\
\text { (Tumaco y Túquerres) }\end{array}$ & $\mathrm{p}=0,014$ & 45 \\
\hline 4 o más motivos EPIYA & $*_{\mathrm{se}}$ & $\mathrm{p}=0,01$ & 49 \\
\hline 3 o más motivos EPIYA-C & Bogotá & $\begin{array}{l}\mathrm{OR}=12,4 \\
2,32-66,3\end{array}$ & 50 \\
\hline gen homB & $*$ se & $\mathrm{p}=0,012$ & 55 \\
\hline gen $j h p 0045$ & $*$ se & $\mathrm{p}=0,015$ & 56 \\
\hline gen $j h p 0046$ & $*_{\mathrm{se}}$ & $\mathrm{p}=0,047$ & 56 \\
\hline
\end{tabular}

*se: sin especificar, por ser población que consultó al Instituto Nacional de Cancerología (Bogotá, Colombia), en donde confluyen pacientes de todas las regiones del país.

\section{REFERENCIAS}

1. Ferlay J, Soerjomataram I, Ervik M, Dikshit $\mathrm{R}$, Eser S, Mathers C, et al. GLOBOCAN 2012 v1.0. Estimated cancer incidence, mortality and prevalence worldwide: IARC Cancer Base No. 11. [Internet]. 2013 [consultada 2013 Oct 20]. Lyon, Francia: Agencia Internacional para la Investigación sobre el Cáncer. Disponible en: http://globocan.iarc.fr

2. Instituto Nacional de Cancerología. El cáncer en cifras [Internet]. 2008 [consultada 2013 Oct 20]. Bogotá, Colombia: Ministerio de Protección Social. Disponible en: http://www.cancer.gov.co/

contenido/categoría.aspx?catID $=434$

3. Daza D. Cáncer gástrico en Colombia entre 2000 y 2009. [Trabajo de Grado en Internet]. Bogotá. Universidad del Rosario; 2012 [consultada 2013 Oct 20]. Disponible en: http://hdl.handle.net/10336/4004

4. Murillo R, Piñeros M, Wiesner C, Rivera $\mathrm{D}$, Bernal L, Aguilera J, et al. Instituto Nacional de Cancerología. Plan Decenal para el Control del Cáncer en Colombia, 2012-2021 [Internet]. 2012 Feb [consultada 2013 Oct 20]. Bogotá, Colombia: Ministerio de Protección Social. Disponible en: http://www.cancer.gov. co/documentos/Plandecenalparaelcon- 
troldelcancer/PlanDecenal_ControlCancer_2012-2021.pdf

5. Vélez-Castrillón S, Camargo JF, Correa PA, Anaya JM. Bases moleculares de la familia de la interleuquina-1. Revista Colombiana de Reumatología. 2004 Mar; 11 (1):11-39.

6. El-Omar EM, Rabkin CS, Gammon MD, Vaughan TL, Risch HA, Schoenberg JB, et al. Increased risk of non cardia gastric cancer associated with proinflamatory cytokine gene polymorphisms. Gastroenterology [Internet]. 2003 May [citado 2013 Jul 04]; 124 (5): 1193-1201. Disponible en http://www.ncbi.nlm.nih. gov/pubmed/12730860

7. Hwang IR, Kodama T, Kikuchi S, Sakai K, Peterson LE, Graham DY, et al. Effect of Interleukin 1 Polymorphisms on Gastric Mucosal Interleukin 1 Production in Helicobacter pylori Infection. Gastroenterology [Internet]. $2002 \mathrm{Dec}$ [citado 2013 Jul 04];123(6): 1793 - 1803. Disponible en: http://www.ncbi.nlm.nih. gov/pubmed/12454835

8. Martínez T, Hernández G, Bravo M, Trujillo E, Quiroga A, Albis R, et al. Asociación de los polimorfismos IL-1B-511 e IL1RN y Helicobacter pylori CagA positivo con cáncer gástrico en una zona de riesgo alto en Colombia. Rev. méd. Chile [Internet]. 2011 Oct [citado 2013 Jul 04]; 139(10): 1313-1321. Disponible en: http:// www.scielo.cl/scielo.php?pid = S003498872011001000010\&script $=$ sci_arttext

9. Martínez T, Hernández G, Bravo M, Trujillo E, Quiroga A, Robayo J, et al. Polimorfismos genéticos de interleucinas IL1B-511, IL-1RN, IL-10, factor de necrosis tumoral alfa-308 e infección por Helicobacter pylori CagA positivo en cáncer gástrico y úlcera duodenal en diferentes poblaciones en Colombia. Rev. colomb. cancerol [Internet]. 2011 Jun [citado
2013 Jul 04]; 15(2): 85-97. Disponible en: http://pesquisa.bvsalud.org/portal/resource/pt/lil-661725

10. Montealegre $M$, Jaramillo $H$, Bohórquez M, Montealegre L, Delgado M. Detección de Helicobacter pylori y caracterización del la región -31 del gen de la Interleucina $1-\beta$ humana en pacientes de una población colombiana con enfermedades gastroduodenales. Rev Col Gastroenterol [Internet]. 2008 Mar [citado 2013 Jul 04]; 23(1): 40-44. Disponible en: http://www.scielo.org.co/scielo. php? script $=$ sci_arttext $\&$ pid $=$ S0120. $99572008000100005 \& \operatorname{lng}=$ es.

11. Arango MT, Jaramillo $C$, Montealegre MC, Bohorquez MH, Delgado MP. Genotipificación de los polimorfismos -511 , -31 y +3954 del gen de la interleucina - IL-1 $\beta$ humana en una población colombiana con cuadro de dispepsia. Biomédica [Internet]. 2010 Apr -Jun [citado 2013 Jul 09]; 30 (2): 199-206. Disponible en: http://www.ncbi.nlm.nih.gov/pubmed/20890567

12. Cañas M, Morán Y, Rivero MB, Bohórquez A, Villegas V, Rendón Y, et al. Polimorfismo genético de interleuquina -1 : Asociación con cáncer gástrico en la población de alto riesgo del Centrooccidente de Venezuela. Rev Med Chil. [Internet]. 2009 Jan [citado 2013 Jul 04];137 (1):63-70. Disponible en: http://www. ncbi.nlm.nih.gov/pubmed/19399323

13. Bayley JP, Ottenhoff TH, Verweij CL. Is there a future for TNF promoter polymorphisms?_Genes Immun [Internet]. 2004 Aug [citado 2013 Jul 04]; 5 (5):315 -29 Disponible en: http://www.ncbi.nlm.nih. gov/pubmed/14973548

14. Machado JC, Figueiredo C, Canedo P, et al. A proinflammatory genetic profile increases the risk for chronic atrophic gastritis and gastric carcinoma. Gastroen-
Junio - Diciembre 2013 ISSN 0122-820X PP: 61-73 
No. 2 terology [Internet]. 2003 Aug [citado 2013 Jul 09];125(2):364-71. Disponible en: http://www.ncbi.nlm.nih.gov/pub$\mathrm{med} / 12891537$

15. Torres MM, Acosta C, Sicard D, Groot H. Susceptibilidad genética y riesgo de cáncer gástrico en una población del Cauca. Biomédica [Internet]. 2004 June [citado 2013 Jul 04]; 24(2):153-162. Disponible en: http:// pesquisa.bvsalud.org/ portal/resource/es/lil-6354139

16. Gorouhi F, Islami F, Bahrami $\mathrm{H}$ and $\mathrm{Ka}$ mangar F. Tumour-necrosis factor-A polymorphisms and gastric cancer risk: a meta-analysis. Br J Cancer [Internet]. 2008 Mar 04 [citado 2013 Jul 03]; 98 (8): 1443 51. Disponible en: http://www.ncbi.nlm. nih.gov/pubmed/ 18319718

17. Shepherd T, Tolbert D, Benedetti J, Macdonald J, Stemmermann G, Wiest J, et al. Alterations in exon 4 of the p53 gene in gastric carcinoma. Gastroenterology [Internet]. 2000 Jun; [citado 2013 Jun 12]; 118 (6): 1039-44. Disponible en: http://www.ncbi.nlm.nih.gov/pub$\mathrm{med} / 10833478$

18. Cardona D, Castaño E, Marín J. Cáncer gástrico, tabaquismo, consumo de licor, estrato socioeconómico y polimorfismo en el codón 72 del gen p53 en una población de Manizales. Biosalud [Internet]. 2007 Ene-Dic [citado 2013 Jun 13]; 6:33- 44. Disponible en: http://pesquisa.bvsalud. org/portal/resource/es/lil-492637

19. Zhou Y, Li N, Zhuang W, Liu G-J, Wu TX, Yao X, et al. p53 codon 72 polymorphism and gastric cancer: A meta-analysis of the literature. Int. J. Cancer [Internet]. 2007 Oct 1 [citado 2013 July 03]; 121 (7): 1481 86. Disponible en: http://www.ncbi.nlm. nih.gov/pubmed/17546594

20. Zhang Q, Ma YY, Wang HJ, Shao CM, Zhang J and Ye ZY. Meta-analysis of the association between P53 codon 72 polymorphisms and gastric cancer. J. Surg.
Oncol [Internet]. 2013 Mar [citado 2013 Jun 18]; 107 (4): 360-6. Disponible en: http://www.ncbi.nlm.nih.gov/pub$\operatorname{med} / 22886602$

21. Setiawan VW, Zhang ZF, Yu GP, Li YL, Lu ML, Tsai CJ, et al. GSTT1 and GSTM1 null genotypes and the risk of gastric cancer: case-control study in a Chinese population. Cancer Epidemiol Biomark Prev [Internet]. 2000 Jan [citado 2013 Jun 18]; 9 (1):73-80. Disponible en: http://www. ncbi.nlm.nih.gov/pubmed/10667466

22. Katoh T, Nagata N, Kuroda Y, Itoh $\mathrm{H}$, Kawahara A, Kuroki N, et al. Gluthation S-transferase M1 (GSTM1) and T1 (GSTT1) genetic polymorphism and susceptibility to gastric and colorectal adenocarcinoma. Carcinogenesis [Internet]. 1996 Sep [citado 2013 Jun 18]; 17(9):1855-9. Disponible en: www.ncbi. nlm.nih.gov/pubmed/8824506

23. Harada S, Misawa S, Nakamura T, Tanaka N, Ueno E., Nozoe M. Detection of GST1 gene deletion by the polymerase chain reaction and its possible correlation with stomach cancer in Japanese. J Hum Genet [Internet]. 1992 Sep - Oct [citado 2013 Jun 18]; 90 (1-2):62-4. Disponible en: http://www.ncbi.nlm.nih.gov/ pubmed/1427788

24. Strange RC, Matharoo B, Faulder GC, Jones P, Cotton W, Elder JB, et al. The human glutathione Stransferase: a case-control study of the incidence of the GST1 0 phenotype in patients with adenocarcinoma. Carcinogenesis [Internet]. 1991 Jan [citado 2013 Jun 18]; 12 (1): 25-8. Disponible en: http://www.ncbi.nlm.nih.gov/ pubmed/1988177

25. Castaño-Molina E, Santacoloma M, Arango L, Camargo M. Cáncer gástrico y genes detoxificadores en una población colombiana. Rev Col Gastroenterol [Internet]. 2010 Jul-Sept [citado 2013 June 19]; 25 (3):252-260. Disponible en: 
http://pesquisa.bvsalud.org/portal/resource/es/lil-589397

26. Georgiadis P, Topinka J, Vlachodimitropoulos D, Stoikidou M, Gioka M, Stephanou G, et al. Interactions between CYP1A1 polymorphisms and exposure to environmental tobacco smoke in the modulation of lymphocyte bulky DNA adducts and chromosomal aberrations. Carcinogenesis [Internet]. 2005 Jan [citado 2013 June 19]; 26 (1):93-101. Disponible en: http://www.ncbi.nlm.nih.gov/ pubmed/15459023

27. Castaño-Molina Eduardo, Santacoloma Mario, Arango Lázaro, Camargo Mauricio. CYP1A1, CYP2E1 y riesgo a cáncer gástrico en una población colombiana de alta incidencia. Acta biol Colomb [Internet]. 2009 Dec [citado 2013 June 19]; 14(3): 205-211. Disponible en: http://www.scielo.org.co/scielo. php? script $=$ sci_arttext \&pid $=$ S0120 $548 \times 2009000300016 \& \operatorname{lng}=$ en.

28. Löfling J, Diswall M, Eriksson S, Bor'en T, Breimer ME and Holgersson J. Studies of Lewis antigens and $H$. pylori adhesion in $\mathrm{CHO}$ cell lines engineered to express Lewis b determinants. Glycobiology [Internet]. 2008 Jul [citado 2013 June 20]; 18 (7): 494-501. Disponible en: http://www.ncbi.nlm.nih.gov/pub$\mathrm{med} / 18400963$

29. Bermúdez Carlos, Insuasty Jesús, Gamarra Germán. Grupo sanguíneo A y riesgo de cáncer gástrico en el Hospital Universitario de Santander (Bucaramanga, Colombia). Acta Med Colomb [Internet]. 2006 Dic [citado 2013 Jun 14] ; 31(4): 400-410. Disponible en: http://www.scielo.org.co/scielo. php? script $=$ sci_arttext $\&$ pid $=$ S0120. $24482006000400003 \& \operatorname{lng}=$ es.

30. You WC, Ma JL, Liu J, Gail MH, Chang Y, Zhang L, et al. Blood type and family cancer history in relation to precancerous gastric lesions. Int J Epidemiol [Internet].
2000 Jun [citado 2013 Jun 20]; 29 (3): 405-7. Disponible en: http://www.ncbi. nlm.nih.gov/pubmed/10869310

Junio - Diciembre 2013 ISSN 0122-820X PP: 61-73

31. de Mattos LC, Rodrigues Cintra J, Sánchez FE, Alves da Silva Rde C, Ruiz MA, Moreira HW. ABO, Lewis, secretor and non-secretor phenotypes in patients infected or uninfected by the Helicobacter pylori bacillus. Sao Paulo Med J [Internet]. 2002 Mar [citado 2013 Jun 20]; 120 (2): 55-8. Disponible en: http://www. ncbi.nlm.nih.gov/pubmed/11994774

32. Shibata A, Hamajima N, Ikehara Y, Saito T, Matsuo K, Katsuda N, et al. ABO blood type, Lewis and Secretor genotypes, and chronic atrophic gastritis: a crosssectional study in Japan. Gastric Cancer [Internet]. 2003 [citado 2013 Jun 20]; 6 (1): 8-16. Disponible en: http://www. ncbi.nlm.nih.gov/pubmed/12673421

33. IARC. Monographs on the evaluation of carcinogenic risks to humans. Schistosomes, liver flukes and Helicobacter pylori. Vol 61. International Agency for Research on Cancer; Lyon, France, 1994.

34. Censini S, Lange C, Xiang Z, Crabtree JE, Ghiara P, Borodovsky M et al. cag, a pathogenicity island of Helicobacter pylori, encodes type I-specific and disease-associated virulence factors. Proc Natl Acad Sci USA [Internet]. 1996 Dec 10 [citado 2013 Jun 20]; 93(25): 14648-53. Disponible en: http://www.ncbi.nlm.nih.gov/ pubmed/8962108

35. Day AS, Jones NL, Lynett JT, Jennings HA, Fallone CA, Beech $\mathrm{R}$ et al. CagE is a virulence factor associated with $\mathrm{He}$ licobacter pylori - induced duodenal ulceration in children. J Infect Dis 2000; 181:1370-5.

36. Backert S, Tegtmeyer N, Selbach M. The versatility of Helicobacter pylori CagA effector protein functions: The master key hypothesis. Helicobacter [Internet]. 2010 
No. 2
Jun [citado 2013 Jun 20]; 15 (3):163176. Disponible en: http://www.ncbi.nlm. nih.gov/pubmed/20557357

37. Huang JQ, Zheng GF, Sumanac K, Irvine EJ, Hunt RH. Meta-analysis of the relationship between $\operatorname{cagA}$ seropositivity and gastric cancer. Gastroenterology [Internet]. 2003 Dec [citado 2013 Jun 20]; 125 (6): 636- 44. Disponible en: http://www. ncbi.nlm.nih.gov/pubmed/14724815

38. Atherton JC. H. pylori virulence factors. Br Med Bull [Internet]. 1998 [citado 2013 Jun 20]; 54 (1):105-20. Disponible en: http://www.ncbi.nlm.nih.gov/pubmed/9604436

39. Yamaoka Y, Kwon DH, Graham DY. AM(r) 34.000 proinflammatory outer membrane proteion (oipA) of Helicobacter pylori. Proc Natl Acad Sci USA [Internet]. 2000 Jun 20 [citado 2013 Jun 20]; 97 (13):7533-8. Disponible en: http://www. ncbi.nlm.nih.gov/pubmed/10852959

40. GerhardM, LehnN, NeumayerN, Boren T, Rad R, Schepp W et al. Clinical relevance of the Helicobacter pylori gene for bloodgroup antigen-binding adhesin. Proc Natl Acad Sci USA [Internet]. 1999 Oct 26 [citado 2013 Jul 10]; 96 (22):12778 83. Disponible en: http://www.ncbi.nlm. nih.gov/pubmed/10535999

41. Cittelly DM, Huertas M, Martínez J, Oliveros $\mathrm{R}$, Posso $\mathrm{H}$, Bravo MM et al. Los genotipos de Helicobacter pylori en gastritis no atrófica difieren de los encontrados en úlcera péptica, lesiones premalignas y cáncer gástrico en Colombia. Rev. méd. Chile [Internet]. 2002 Feb [citado 2013 Jun 20]; 130(2): 143-151. Disponible en: http://www.scielo.cl/scielo. php? script $=$ sci_arttext $\&$ pid $=\mathrm{S} 0034$. $98872002000200003 \& \operatorname{lng}=$ es . http://dx.doi.org/10.4067/S003498872002000200003.

42. Quiroga AJ, Cittelly DM, Bravo MM. Frecuencia de los genotipos babA2, oipA y cagE de Helicobacter pylori en pacientes colombianos con enfermedades gastroduodenales. Biomédica [Internet]. 2005 Sep [citado 2013 Jun 22]; 25(3):325-334. Dis p o n i ble en: http://www.scielo.org.co/scielo. php? script $=$ sci_arttext\&pid $=$ S0120 $41572005000300008 \& \operatorname{lng}=$ en .

43. Mizushima T, Sugiyama T, Komatsu Y, Ishizuka J, Kato M, Asaka M. Clinical Relevance of the babA2 genotype of Helicobacter pylori in Japanese Clinical Isolates. J. Clin. Microbiol [Internet]. 2001 July [citado 2013 Jun 22]; 39 (7) 2463-5. Disponible en: http://www.ncbi.nlm.nih. gov/pubmed/11427555

44. López AM, Delgado MP, Jaramillo C, Amézquita A, Parra G, Echeverry MM. Caracterización del gen de la citotoxina vacuolizante de Helicobacter pylori a partir de biopsias gástricas de pacientes residentes en Tolima, Colombia. Rev. argent. microbiol. [Internet]. 2009 Ene-Mar [citado 2013 Jul 03]; 41 (1) pp. 4-10. Disponible en: http://pesquisa.bvsalud.org/ portal/resource/es/lil-634609

45. Loh JT, Shaffer CL, Piazuelo MB, Bravo LE, McClain MS, Correa P, et al. Analysis of cagA in Helicobacter pylori strains from Colombian populations with contrasting gastric cancer risk reveals a biomarker for disease severity. Cancer Epidemiol Biomarkers Prev. [Internet] 2011 Oct [citado 2013 Jul 09]; 20 (10):223749. Disponible en: http://www.ncbi.nlm. nih.gov/pmc/articles/PMC3189306/

46. Higashi H, Tsutsumi R, Fujita A, Yamazaki S, Asaka M, Azuma T, Hatakeyama M. Biological activity of the Helicobacter pylori virulence factor CagA is determined by variation in the tyrosine phosphorylation sites. Proc Natl Acad Sci USA [Internet] 2002 Oct 29 [citado $2013 \mathrm{Jul}$ 09]; 99(22):14428-33. Disponible en: http://www.ncbi.nlm.nih.gov/pubmed/ PMC137900/ 
47. Argent RH, Kidd M, Owen RJ, Thomas RJ, Limb MC, Atherton JC. Determinants and consequences of different levels of CagA phosphorylation for clinical isolates of Helicobacter pylori. Gastroenterology [Internet] 2004 Aug [citado 2013 Jul 09]; 127(2):514-23. Disponible en: http://www.ncbi.nlm.nih.gov/ pubmed/15300584

48. Azuma T, Yamakawa A, Yamazaki S, Fukuta K, Ohtani M, Ito Y, et al. Correlation between variation of the 3' región of the CagA gene in Helicobacter pylori and disease outcome in Japan. J Infect Dis [Internet] 2002 Dec 01 [citado 2013 Jul 09];186 (11):1621-30. Disponible en: http://www.ncbi.nlm.nih.gov/pub$\mathrm{med} / 12447739$

49. Schneider N, Krishna U, Romero-Gallo J, Israel DA, Piazuelo MB, Camargo MC, et al. Role of Helicobacter pylori CagA Molecular Variations in Induction of Host Phenotypes with Carcinogenic Potencial. J Infect Dis [Internet] 2009 April 15 [citado 2013 Jul 09]; 199(8):1218-1221. Disponible en: http://www.ncbi.nlm.nih. gov/pubmed/19278338

50. Quiroga AJ, Huertas A, Cómbita AL, Bravo MM. Variación en el número de repeticiones EPIYA-C en la proteína CagA de aislamientos colombianos de Helicobacter pylori y su capacidad para inducir fenotipo colibrí en células epiteliales gástricas. Biomédica [Internet] 2010 Jun [citado 2013 Jun 22]; 30(2):251-258. Disponible en: http://pesquisa.bvsalud. org/portal/resource/es/lil-560973

51. Fajardo CA, Quiroga AJ, Coronado A, Labrador K, Acosta N, Delgado P, et al. CagA EPIYA polymorphisms in Colombian Helicobacter pylori strains and their influence on disease-associated cellular responses. World J Gastrointest Oncol. [Internet] 2013 March 15 [citado 2013 Jun 22]; 5(3):50-59. Disponible en: http://www. ncbi.nlm.nih.gov/pubmed/23671731
52. Torres KE, Moran YH, Valderrama EJ, Chiurillo MA. Variantes del motivo EPIYA de la proteína CagA de Helicobacteri pylori en biopsias gástricas de pacientes con gastritis crónica de la región centrooccidental de Venezuela. Revista de la Sociedad Venezolana de Microbiología [Internet] 2013 [citado 2013 June 22]; 33:18-23. Disponible en: http://www. scielo.org.ve/pdf/rsvm/v33n1/art05.pdf

53. Oleastro M, Cordeiro R, Ferrand J, Nunes B, Lehours P, Carvalho-Oliveira I, et al. Evaluation of the clinical significance of homB, a novel candidate marker of Helicobacter pylori strains associated with peptic ulcer disease. J. Infect. Dis. [Internet] 2008 Nov 01 [citado 2013 Jun 22]; 198 (9):1379-1387. Disponible en: http://www.ncbi.nlm.nih.gov/pubmed/18811585

54. Oleastro M, Monteiro L, Lehours P, Megraud $\mathrm{F}$ and $\mathrm{A}$. Menard. Identification of markers for Helicobacter pylori strains isolated from children with peptic ulcer disease by suppressive subtractive hybridization. Infect. Immun. [Internet] $2006 \mathrm{Jul}$ [citado 2013 Jun 22]; 74 (7):4064-4074. Disponible en: http://www.ncbi.nlm.nih. gov/pubmed/16790780

55. Jung SW, Sugimoto M, Graham DY, Yamaoka Y. homB status of Helicobacter pylori as a novel marker to distinguish gastric cancer from duodenal ulcer. J Clin Microbiol. [Internet] 2009 Oct [citado 2013 Jul 09]; 47(10): 3241-3245. Disponible en: http://www.ncbi.nlm.nih.gov/ pubmed/19710266

56. Watada M, Shiota S, Matsunari O, Suka Y. Association between Helicobacter pylori cagA-related genes and clinical outcomes in Colombia and Japan. BMC Gastroenterol. [Internet] 2011 Dec 22 [citado 2013 Jun 22]; 11:141. Disponible en: http://www.ncbi.nlm.nih.gov/pub$\mathrm{med} / 22189161$

Junio - Diciembre 2013

ISSN 0122-820X

PP: 61-73 